\title{
Measurement of Interleukin-6 Levels in COVID: Illuminative or Illogical?
}

\author{
Ashit Hegde 10 \\ Keywords: COVID-19 mortality, Interleukin-6 receptor antagonists, Interleukin-6. \\ Indian Journal of Critical Care Medicine (2022): 10.5005/jp-journals-10071-24102
}

Interleukin-6 (IL-6), which was first discovered in 1973 by researchers at the Osaka University, is a cytokine with multiple effects. It plays a role in immune regulation, hematopoiesis, inflammation and oncogenesis. ${ }^{1}$ Several pathological conditions including infection, inflammation, and cancer stimulate the release of IL- 6 from macrophages, mast cells and dendritic cells. Endothelial cells, epithelial cells, and fibroblasts also express IL-6 in response to specific stimuli.

IL- 6 is considered to be the key mediator of the unregulated proinflammatory response which is supposed to be the prime contributor to lung damage in patients with severe COVID. ${ }^{2}$

Several studies and meta-analyses have demonstrated a clear association between high IL- 6 levels and an increased severity of COVID-19. ${ }^{3,4}$ Studies examining the link between IL- 6 levels and mortality have had conflicting results, however.

The study in this issue by Talwar et al. ${ }^{5}$ concluded that the measurement of IL-6 levels on admission to intensive care unit (ICU) could not be used to predict mortality. A recent meta-analysis by Liu et al. also concluded that though IL- 6 levels predicted severity of disease, they were not reliable predictors of mortality. ${ }^{6}$

Most of the studies which found a link between IL- 6 levels and mortality measured IL- 6 levels on admission to hospital. These patients had varying severity of illness at the time of measurement. Whereas in this study by Talwar et al., IL- 6 levels were measured only after the patients became sick enough to need intensive care and it is possible that IL- 6 becomes a less reliable indicator when measured in a smaller group of severely ill patients.

It must also be understood that IL- 6 levels tend to fluctuate in an individual during the day and this might also contribute to the inconsistent results.

Some studies therefore suggest that serial levels of IL- 6 might be more useful than an isolated value. Rising levels or a sudden peak in levels of IL- 6 might be more accurate predictors of mortality. ${ }^{8}$

The proinflammatory actions of IL- 6 may not be directly related to its serum levels. Its actions may depend on the balance between the levels of the pro- and anti-inflammatory cytokines. The levels of circulating IL- 6 receptors also influence the action of IL- $6 .{ }^{9}$

Some studies have claimed that combining the levels of other cytokines along with IL- 6 levels might be superior at predicting mortality. ${ }^{10}$

It is also interesting to note that in this study by Talwar et al., the other markers of severity-the NL ratio and CT severity scores correlated with IL-6 levels and by inference probably did not correlate with mortality. These correlations were not studied, however.

It is likely therefore that while inflammation might contribute to the severity of illness, several factors other than inflammation alone might be responsible for mortality.
PD Hinduja Hospital, Mumbai, Maharashtra, India

Corresponding Author: Ashit Hegde. PD Hinduja Hospital, Mumbai, Maharashtra, India, Phone: +91 22 24462250, e-mail: ahegde1957@ gmail.com

How to cite this article: Hegde A. Measurement of Interleukin-6 Levels in COVID: Illuminative or Illogical? Indian J Crit Care Med 2022;26(1): 9-10.

Source of support: Nil

Conflict of interest: None

On the basis of their findings, the authors surmised that IL- 6 receptor antagonists (IL-6ra) might not be useful in the treatment of COVID-19. The World Health Organization Rapid Evidence Appraisal for COVID Therapies (REACT) working group has published a meta-analysis which belies this assumption. ${ }^{11}$

The REACT group analyzed 27 randomized controlled trials (RCTs) of IL-6ra and concluded that IL-6ra reduced mortality in severely ill patients with COVID-19. The benefits were seen only in patients who had also received steroids.

A few small studies have concluded that IL-6ra is most beneficial in patients with high IL- 6 levels. ${ }^{12}$ The correlation (if any) between IL- 6 levels and benefit of IL- 6 ra was not addressed in this meta-analysis, however. The REACT group, however, concluded that the benefit of IL-6ra was not linked to C-reactive protein (which might be a surrogate for IL-6) levels. Whether measurement of IL- 6 levels (either on admission to the ICU or serially) will help select a sub group of patients who are more likely to respond to IL- 6 antagonists needs to be studied in a larger group of patients.

Measurement of IL-6 levels is not easily possible in a majority of centers in our country. There can be errors in the measurement if the blood sample is not processed quickly. Evidence available until now suggests that the benefit of IL-6ra probably does not correlate with IL- 6 levels. Therefore, once a patient has reached the ICU, there is probably no rationale for measuring IL-6 levels either to predict mortality or to select patients for IL-6ra therapy.

\section{OrCID}

Ashit Hegde (으 https://orcid.org/0000-0003-4342-122X

\section{References}

1. Kishimoto T. IL-6: from its discovery to clinical applications. Int Immunol 2010;22(5):347-352. DOI: 10.1093/intimm/dxq030.

2. Coomes EA, Haghbayan H. Interleukin- 6 in Covid-19: a systematic review and meta-analysis. Rev Med Virol 2020;30(2):e2141. DOI: 10.1002/rmv.2141. 
3. Liu X, Shi S, Xiao J, Wang H, Chen L, Li J, et al. Prediction of the severity of the coronavirus disease and its adverse clinical outcomes. Jpn J Infect Dis 2020;73(6):404-410. DOI: 10.7883/yoken.JJID. 2020.194.

4. Henry BM, De Oliveira MHS, Benoit S, Plebani M, Lippi G. Hematologic, biochemical and immune biomarker abnormalities associated with severe illness and mortality in coronavirus disease 2019 (COVID-19): a meta-analysis. Clin Chem Lab Med (CCLM) 2020;58(7):1021-1028. DOI: 10.1515/cclm-2020-0369.

5. Talwar D, Kumar S, Acharya S, Raisinghani N, Madaan S, Hulkoti V, et al. Interleukin 6 and Its Correlation with COVID-19 in Terms of Outcomes in an Intensive Care Unit of a Rural Hospital: A Crosssectional Study. Indian J Crit Care Med 2022;26(1):39-42.

6. Liu X, Wang H, Shi S, Xiao J. Association between IL-6 and severe disease and mortality in COVID-19 disease: a systematic review and meta-analysis. Postgrad Med J 2021; postgradmedj-2021-139939. DOI: 10.1136/postgradmedj-2021-139939.

7. Nilsonne G, Lekander M, Åkerstedt T, Axelsson J, Ingre M. Diurnal variation of circulating interleukin- 6 in humans: a meta-analysis. PLoS One 2016;11(11):e0165799. DOI: 10.1371/journal.pone. 0165799.
8. Chen X, Zhou J, Chen C, Hou B, Ali A, Li F, et al. Consecutive monitoring of interleukin- 6 is needed for COVID-19 patients. Virol Sin 2021;36(5):1093-1096. DOI: 10.1007/s12250-021-00425-4.

9. Jostock T, Müllberg J, Özbek S, Atreya R, Blinn G, Voltz N, et al. Soluble gp130 is the natural inhibitor of soluble interleukin- 6 receptor transsignaling responses. Eur J Biochem 2001;268(1):160-167. DOI: 10.1046/j.1432-1327.2001.01867.x.

10. Dorgham K, Quentric P, Goekkaya M, Marot S, Parizot C, Sauce D, et al. Distinct cytokine profiles associated with COVID-19 severity and mortality. J Allergy Clin Immunol 2021;147(6):2098-2107. DOI: 10.1016/j.jaci.2021.03.047.

11. The WHO Rapid Evidence Appraisal for COVID-19 Therapies (REACT) Working Group, Shankar-Hari M, Vale CL, Godolphin PJ, Fisher D, Higgins JPT, Spiga F, et al. Association between administration of IL-6 antagonists and mortality among patients hospitalized for COVID19: a meta-analysis. Journal of the American Medical Association 2021;326(6):499-518. DOI: 10.1001/jama.2021.11330.

12. Galvan-Roman JM, Rodriguez-Garcia SC, Roy-Vallejo E, et al. IL-6 serum levels predict severity and response to tocilizumab in COVID-19: an observational study. J Allergy Clin Immunol 2020;147(1):72-80. DOI: 10.1016/j.jaci.2020.09.018. 